\section{Orthogeriatrics in Italy: the Gruppo Italiano di Ortogeriatria (GIOG) audit on hip fractures in the elderly}

\author{
Amedeo Zurlo, ${ }^{1}$ Giuseppe Bellelli \\ on behalf of GIOG* \\ ${ }^{1}$ Department of Medical Sciences \\ University of Ferrara; Geriatrics and \\ Orthogeriatric Unit, University Hospital \\ of Ferrara; ${ }^{2}$ School of Medicine and \\ Surgery, Milano-Bicocca University \\ Milano; Orthogeriatric Unit, S. Gerardo \\ Hospital, Monza (MB), Italy
}

\begin{abstract}
The Gruppo Italiano di Ortogeriatria (GIOG) is an Italian study group promoted by three Italian Scientific Geriatric Societies with the aim of disseminating orthogeriatric methodology in Italy. In 2015 it has supported a multicenter web-based audit to collect data on functioning of Italian orthogeriatrics. The study, still ongoing, has enrolled until now 2577 cases of elderly patients undergoing surgery for hip fracture from 14 Italian orthogeriatric units. The population in question consists of markedly elderly and frail subjects, due to high prevalence of pre-existing functional deficit conditions, but it is also clinically complex; the most frequent fracture is intertrochanteric and the most performed surgery approach is intramedullary nail. This is the largest multicenter observational study conducted so far in Italy on elderly patients with hip fracture.
\end{abstract}

\section{Introduction}

Hip fracture is a major public health issue worldwide ${ }^{1}$ and in Italy too. ${ }^{2,3}$ Data obtained from the 2017 annual report of National Health Ministry Programma Nazionale Esiti (PNE 2017) indicate that in 2016 more than 123,000 patients with hip fracture were admitted to hospital in Italy; of these more than 7000 patients have dyed within the first month. ${ }^{4}$ The same epidemiological data indicate a forecast of deaths within 1 year from the injury event greater than 22,000, while it is estimated that at 1 year from the fracture event about 50,000 of these patients will have developed a variable degree of motor disability. Considering these data together with the high prevalence of comorbidity and peri-operative medical complications in this population, it is neces- sary to interpret hip fracture as a complex syndrome that must be addressed in a multidisciplinary, multi-professional and multisetting approach requiring a comprehensive methodology for which Geriatrician, by vocation, expertise and competence, certainly appears as the reference figure.

Orthogeriatrics has given evidence to represents the methodology able to obtain significant advantages in terms of outcomes on fractured elderly patients and on health organizations, ${ }^{5-7}$ through different intervention models but anyway a comanagement approach of patients, together with Orthopedics and all other specialists involved in hip fracture clinical/care pathway, is essential

The Gruppo Italiano di Ortogeriatria (GIOG) is an Italian inter-company study group promoted by Società Italiana $d i$ Gerontologia e Geriatria (SIGG) together with Associazione Italiana di Psicogeriatria (AIP) and Società Italiana di Geriatria Ospedale e Territorio (SIGOT). It has been established in 2012 to be the place of comparison between various national orthogeriatrics experiences and has the basic purpose of disseminating orthogeriatric methodology in order to obtain the highest levels of effectiveness in dealing hip fracture syndrome in the elderly. GIOG has formalized and published in 2014 its national guidelines on hip fracture in the geriatric patient ${ }^{8}$ and promoted in 2016 a web-based audit among centers belonging to the GIOG entitled Register of hip fractures in the GIOG Italian Orthogeriatric Units, with the purpose of obtaining preliminary data on functioning of Italian orthogeriatrics through the establishment of a national level database.

The study, authorized by Ethics Committee of the promoter center and in accordance with current rules on informed consent and privacy, aims to create a centralized computerized database to make all data of participating centers uniform and in digital format; access is protected by a password, no personal data are entered allowing identification of the subject.

\section{Materials and Methods}

The database includes all patients aged $\geq 65$ years undergoing surgery for hip fracture, available to participate in the study (informed consent from the patient or a relative or caregiver) and able to speak Italian language. It consists of data collection modules (Figure 1) focused on epidemiological aspects, clinical data and effectiveness markers, included an optional telephonic follow-up at 30 and 120 days after surgery.
Correspondence: Amedeo Zurlo, Department of Medical Sciences, University of Ferrara, U.O. Geriatrics and Orthogeriatrics,

University Hospital of Ferrara, via A. Moro 8, 44124 Ferrara, Italy. Tel.: +39.0532.236009 -

Fax: +39.0532 .239817$

E-mail: a.zurlo@ospfe.it

Key words: Orthogeriatrics; hip fracture; elderly; co-management; audit.

Conflict of interest: the authors declare no potential conflict of interest.

Conference presentation: $32^{\text {nd }}$ National Congress of Società Italiana di Geriatria Ospedale e Territorio (SIGOT), 3-5 May 2018, Roma, Italy.

Received for publication: 31 July 2018. Accepted for publication: 31 August 2018.

This work is licensed under a Creative Commons Attribution-NonCommercial 4.0 International License (CC BY-NC 4.0).

(C) Copyright A. Zurlo et al., 2018

Licensee PAGEPress, Italy

Geriatric Care 2018; 4:7726

doi:10.4081/gc.2018.7726

14 Italian orthogeriatric units have joined the study, still underway, and in March 2018 the database has recorded 2577 hip fracture cases. Descriptive analysis of collected data allows verifying some important findings.

\section{Results and Discussion}

Hip fracture predominantly occurs in women $(76.3 \%)$, the average age of patients is particularly high $(84.8$ aa \pm 7$)$ and $91.3 \%$ of fractured patients were in their own home at the moment of the injury. $55.5 \%$ of the patients already had some difficulties in gait and pre-existing disabilities before fracture and the degree of clinical complexity is consistent: $66.3 \%$ of patients is ranked as $\geq 3$ class by American Society of Anesthesiologists (ASA) Classification. ${ }^{9}$ Available data are strongly indicative of how hip fracture in the elderly does not represent a purely accidental event in patient's clinical history, but instead constitutes the epiphenomenon of a frailty condition and clinical vulnerability started long time before the fracture. ${ }^{10}$ On the other hand database analysis shows that these patients are usually not subjected to an effective osteoporotic therapeutic prevention strategy, since it appears that only a small percentage of fractured subjects are treated with drugs or nutritional sup- 
plements for primary prevention of osteoporotic fragility fractures $(86.4 \%$ of the fractured patients does not take any specific therapeutic principle). A further confirmation of functional frailty in hip fracture older patients is that about $50 \%$ of cases already present some degree of cognitive impairment at the time of hospitalization, evaluated as $\geq 5$ score at Short Performance Mental Status Questionnaire (SPMSQ), ${ }^{11}$ thereby laying a solid starting point for development of typical postoperative complications such as delirium. The prevailing fracture is intertrochanteric $(39.1 \%)$, followed by displaced intracapsular (14.9\%), undisplaced intracapsular $(14.9 \%)$ and at last subtrochanteric (8\%). The most common type of surgical approach is intramedullary nail $(46.2 \%)$ compared to hemiarthroplasty (30.1), total hip replacement $(9.5 \%)$, sliding hip screw $(6.2 \%)$ and osteosynthesis with cannulated screw (5.9\%).

Regarding timings, average timing of surgery is 2.4 days $(54 \mathrm{~h})$, substantially in agreement with national Italian average in pre-operative timing reported by PNE 2017 data; average length of hospital stay in Orthopedics is substantially limited: 8 days from the intervention, 11 days from admission.

Markers of postoperative care show that at least $1 / 3$ of the cases do not receive allow of fully weight bear without restriction on operated limb in the immediate postoperative period (the day after); prevalence of delirium, about $24 \%$, is within the range of variability present in literature, while the percentage of patients starting rehabilitation the day after surgery $(12.6 \%)$ is not adequate at all.

It should also be noted that protein supplementation, an other marker of good clinical practice reported in literature, is administered minimally $(3.3 \%$ of the overall case series) and that removal of bladder catheter is often carried out late (95\% of patients keep Foley in first day post-op), while the appearance of pressure lesions for the entire length of hospitalization is rather limited, affecting only $3.5 \%$ of patients. Although osteoporosis medical treatment is strongly recommended after hip fracture, only $30 \%$ of patients are discharged from hospital with a multifactorial bone-strengthening treatment for secondary prevention of fragility fractures .

An additional interesting feedback derived from database analysis is represented by comparison of efficacy indicators among participating centers in the audit. In this respect, the most significant data concern timing of surgery and the allocation of patient after discharge from Orthopedics, which represent a considerable heterogeneity among various orthogeriatrics units (Figures 2 and 3).

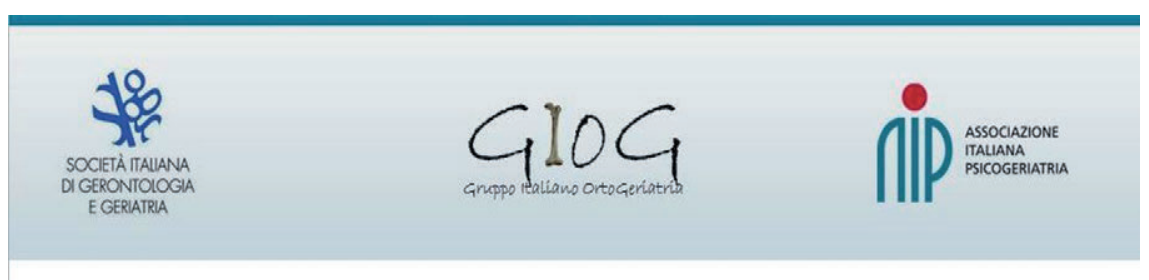

\section{FRAGILITY FRACTURE NETWORK: GRUPPO DEDICATO AL REGISTRO FRATTURA DI FEMORE}
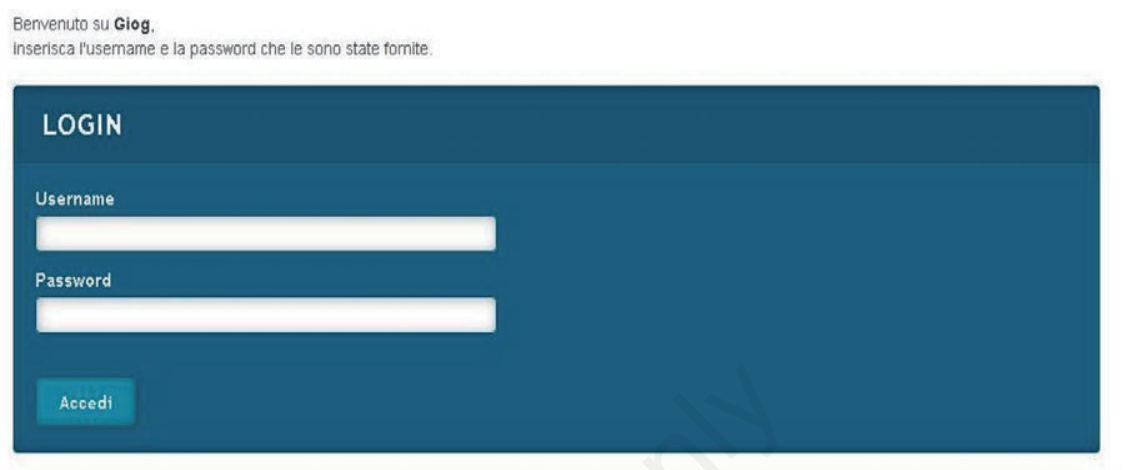

Figure 1. Web-portal of GIOG audit.

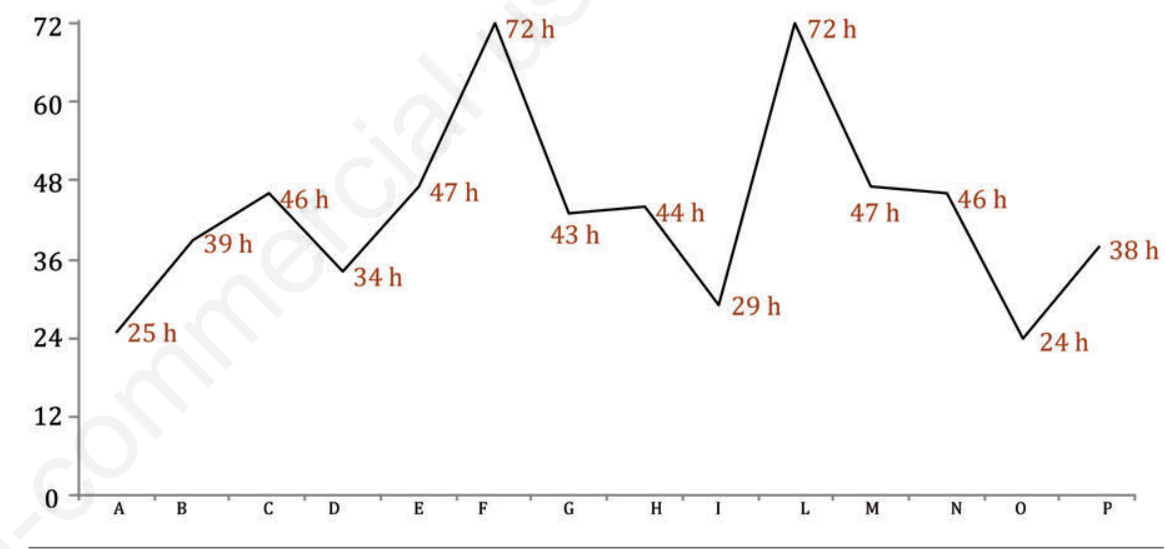

Figure 2. Average time to surgery in GIOG audit.

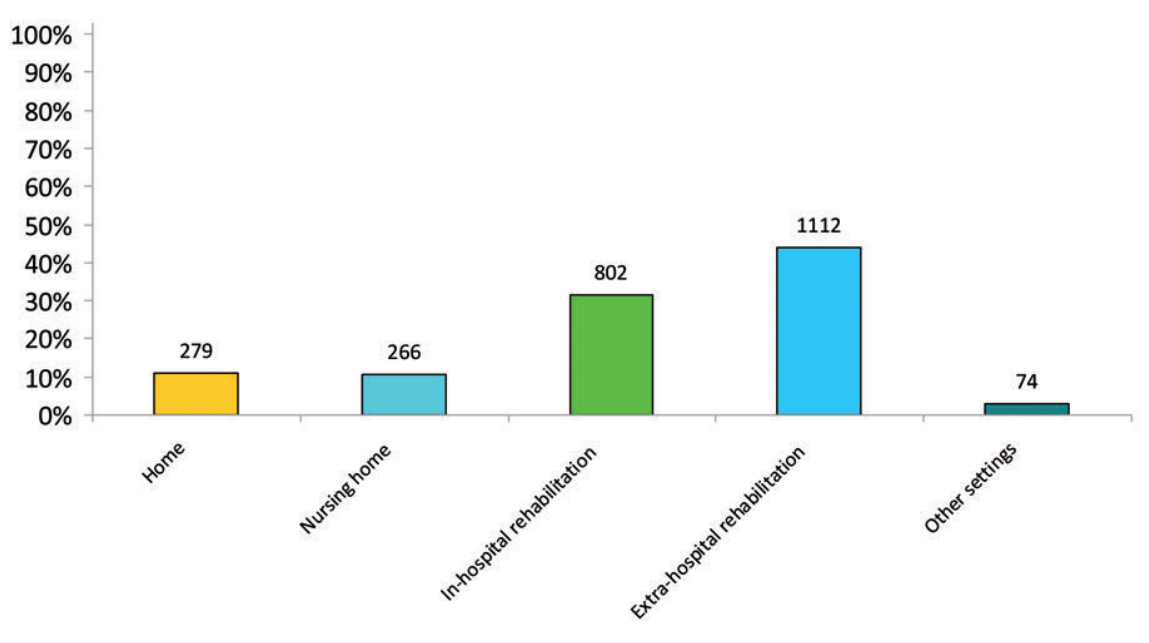

Figure 3. Patient allocation after orthopedic discharge. 
The same consideration can be expressed about anesthetic approach, especially in regards to a significant tendency to recourse to general anesthesia or to regional nerve block techniques only in few centers compared to overall average in the whole sample.

Particular significance must be lastly reserved to discharge planning from hospital and post-hospital care. Data obtained from the analysis of the optional telephonic 30-days and 120-days follow-up after hospital discharge (395 patients) show the frequent beginning of a multi-setting pathway of intermediate care (on average from 3 to 5 alternative settings) before final allocation in the definitive living environment. That justifies need for greater attention to quality and appropriateness of care intervention in these transitional settings, which generally lack of data on activities and outcomes as well as effectiveness.

\section{Conclusions}

In conclusion, the ongoing multi-center GIOG audit on Italian orthogeriatric units has opened a window on a still little-known context in this country and has showed some important aspects that deserve particular consideration. The population in question consists of markedly elderly and frail subjects, due to high prevalence of preexisting functional deficit conditions and limited physiologic reserves, but it's also significantly complex from a clinical point of view. Some aspects of good clinical practice have been highlighted but there are still few indicators of effectiveness not yet adequately appropriate in national orthogeriatric models and it is furthermore detectable a considerable variability among Italian orthogeriatric units outcomes, almost to indicate a partial tendency only to a systematized approach towards quality standards of care emphasized in guidelines and in literature. ${ }^{12,13}$
Hospitalized hip fracture elderly patient has such peculiarities to require a specific approach, in which the orthogeriatric method, based on comprehensive evaluation and management, continuity of care and multidisciplinary, has shown to produce better outcomes than a traditional model of orthopedic care. ${ }^{14}$

Orthogeriatric care is the first and most structured attempt to disseminate a multidimensional and multidisciplinary approach to elderly patients in a multisetting clini$\mathrm{cal} /$ care pathway not exclusively within the competence of the Geriatrician. Through a comanagement methodology it is able to implement a concept of Medicine focused on patient rather on pathology or setting of hospitalization. Data produced by the GIOG audit represent the first phase of analysis of functioning of the national hip fracture care system and are an unavoidable premise for debate on future projects aimed at improving quality of overall clinical practice and care pathways for hip fracture older patients in Italy.

\section{References}

1. Holt G, Smith R, Duncan K, et al. Changes in population demographics and the future incidence of hip fracture. Injury 2009;40:722-6.

2. Piscitelli P, Brandi ML, Tarantino U, et al. Incidenza e costi delle fratture di femore in Italia: studio di estensione 2003-2005. Reumatismo 2010;62:113-8.

3. Piscitelli P, Iolascon G, Argentiero A, et al. Incidence and costs of hip fractures vs strokes and acute myocardial infarction in Italy: comparative analysis based on national hospitalization records. Clin Interv Aging 2012;7:575-3.

4. Italian Health Ministry-National Agency for Regional Health Services. Programma Nazionale Esiti (PNE): PNE Report 2017. Available from: http://pne2017.agenas.it/
5. Friedman SM, Mendelson DA, Kates SL. Geriatric comanagement of proximal femur fractures: total quality management and protocol-driven care result in better outcomes for a frail patient population. J Am Geriatr Soc 2008;56:1349-56.

6. Giusti A, Barone A, Razzano M, et al. Optimal setting and care organization in the management of older adults with hip fracture. Eur J Phis Rehabil Med 2011;47:281-96.

7. Grigoryan KV, Javedan H, Rudolph JL. Orthogeriatrics care models and outcomes in hip fracture patients: a systematic review and meta-analysis. J Ortop Trauma 2014;28:49-55.

8. Pioli G, Barone A, Mussi C, et al. The management of hip fracture in the older population. Joint position statement by Gruppo Italiano Ortogeriatria (GIOG). Aging Clin Exp Res 2014;26:547-53.

9. Kilgore ML, Curtis JR, Delzell E, et al. A close examination of healthcare expenditures related to fractures. J Bone Miner Res. 2013;28:816-20.

10. Owens WD, Felts JA, Spitznagel EL, Jr. ASA physical status classifications: a study of consistency of ratings. Anesthesiology 1978;49:239-43.

11. Pfeiffer E. A short portable mental status questionnaire for the assessment of organic brain deficit in elderly patients. J Am Geriatr Soc 1975;23:433-41.

12. National Clinical Guideline Centre. The management of hip fracture in adults. London, UK: National Clinical Guideline Centre; 2011. Available from: https://www.nice.org.uk/guidance/cg124

13. Scottish Intercollegiate Guidelines Network. Management of hip fracture in older people: a national clinical guideline. Edinburgh, UK: Sign; 2009. Available from: http:/www.sign.ac.uk/ pdf/sign

14. Neuburger J, Currie C, Wakeman R, et al. Increased orthogeriatrician involvement in hip fracture care and its impact on mortality in England. Age Ageing 2017;46:187-92.

\section{*List of GIOG Audit Contributors:}

Tassistro E. (School of Medicine and Surgery, Milano-Bicocca University, Milano); Rapazzini P. (Orthogeriatric Unit, Circolo Hospital, Varese); Mussi C. (Orthogeriatric Unit, University of Modena and Reggio Emilia, Modena); Corsi M. (Orthogeriatric Unit, S. Gerardo Hospital, Monza); Lunardelli M.L. (Orthogeriatric Unit, S. Orsola Malpighi, Bologna); Andreano A. (School of Medicine and Surgery, Milano-Bicocca University, Milano); Castoldi G. (Orthopedic Unit, ASST Vimercate, Carate Brianza Hospital, Vimercate, MB); Floris P. (Orthogeriatric Unit, Hospital of Sondrio-ASST VAL, Sondrio); Pizzonia M. (Orthogeriatric Unit, San Martino Hospital, Genova); Barone A. (Orthogeriatric Unit, Galliera Hospital, Genova); March A. (Orthogeriatric Unit, Bolzano Hospital, Bolzano); Ungar A. (Geriatrics and Intensive Care Unit, Firenze); Galmarini V. (Orthopedic Unit, ASST Fatebenefratelli Sacco, Milano); Franzoni S., Ranieri P. (Department of Medicine and Rehabilitation, S. Anna Hospital, Brescia); Mantovani G., Govoni B. (Geriatrics and Orthogeriatric Unit University Hospital of Ferrara). 